\title{
PomGnT1 enhances temozolomide resistance by activating epithelial-mesenchymal transition signaling in glioblastoma
}

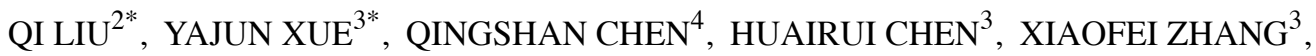

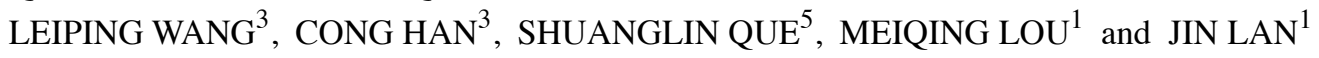

\author{
${ }^{1}$ Department of Neurosurgery, Ren Ji Hospital, School of Medicine, Shanghai Jiao Tong University, Shanghai; \\ ${ }^{2}$ Department of Neurosurgery, Jingdezhen Second Hospital, Jingdezhen, Jiangxi; ${ }^{3}$ Department of Neurosurgery, \\ General Hospital, Shanghai; ${ }^{4}$ Department of Neurosurgery, The Second People's Hospital of Liaocheng City, Liaocheng, \\ Shandong; ${ }^{5}$ Department of Neurosurgery, Longyan First Hospital, Fujian Medical University, Longyan, Fujian, P.R. China
}

Received February 27, 2017; Accepted July 28, 2017

DOI: 10.3892/or.2017.5964

\begin{abstract}
Temozolomide (TMZ) is commonly used in glioblastoma (GBM) chemotherapy. However, a great challenge for TMZ treatment is the rapid development of resistance and subsequent tumor recurrence and poor outcome. In the present study we established TMZ-resistant GBM cells (U87-TR and U251-TR) and found that the expression of PomGnT1 was significantly upregulated in TMZ-resistant GBM cells compared with the TMZ-sensitive counterparts. Furthermore, overexpression of PomGnT1 in U87-MG and U251-MG cells led to increased IC $_{50}$ values for TMZ and reduced apoptosis of cells. Knockdown of PomGnT1 in both U87-TR and U251-TR cells led to decreased $\mathrm{IC}_{50}$ values for $\mathrm{TMZ}$ and enhanced apoptosis. Biochemical analysis revealed that PomGnT1 regulates the expression of factors in epithelial-mesenchymal transition signaling including TCF8, vimentin, $\beta$-catenin and Slug in GBM cells. These findings demonstrate that PomGnT1 might be a new focus of GBM research for treatment of recurrent TMZ-resistant GBM.
\end{abstract}

\section{Introduction}

Glioblastoma (GBM) is among the least curable types of cancer with a median survival of approximately 15 months (1-3). Because of the high infiltrative character of GBM, tumor cells cannot be completely removed through surgical resection or irradiation $(4,5)$. Thus, temozolomide (TMZ), which is one of the most important agents of current standard adjuvant chemotherapy for GBM, aims to abolish these residual tumor cells (6-8). However, resistance to

Correspondence to: Dr Jin Lan, Department of Neurosurgery, Ren Ji Hospital, School of Medicine, Shanghai Jiao Tong University, Shanghai 200127, P.R. China

E-mail: lanjinh@126.com

${ }^{*}$ Contributed equally

Key words: glioma, temozolomide, PomGnT1, drug resistance, apoptosis temozolomide often develops quickly and results in tumor recurrence and poor outcome (9-11). Therefore, understanding the mechanisms of TMZ resistant in GBM cells is essential for the GBM treatment.

O-mannose-b-1,2-N-acetylglucosaminyltransferase (PomGnT1), a glycosyltransferase that participates in the formation of GlcNAc- $\beta 1,2-$ Man glycan, is causally related to muscle-eye-brain disease (MEB), a congenital muscular dystrophy (12). Our earlier studies showed that PomGnT1 expression in GBM tissues was closely associated with poor prognosis in GBM patients and PomGnT1 promoted GBM progression via activation of $\beta$-catenin $(13,14)$. The pathological function of PomGnT1 in GBM is in control of cell adhesion and migration abilities (15-17). However, the function and underlying mechanisms of PomGnT1 in TMZ resistance in GBM have not been reported.

Epithelial-to-mesenchymal transition (EMT) is a process initially observed in embryonic development in which cells lose epithelial characteristics and gain mesenchymal properties to increase motility and invasion, and this process is also important in tumor progression and metastasis (18-20). Recent studies found that EMT was closely related to chemo-resistance (21-23). Blocking the EMT pathway abrogated resistance to anti-folate chemotherapy in lung cancer $(24,25)$.

In the present study, we determined the expression level of PomGnT1 in TMZ-resistant GBM cells, and explored the biological function and potential mechanism of PomGnT1 in TMZ resistant GBM cells.

\section{Materials and methods}

Materials. TMZ and all other reagents were provided by SigmaAldrich (St. Louis, MO, USA). TMZ was diluted in dimethyl sulfoxide (DMSO; Beijing Solarbio Science and Technology, Co., Ltd., Beijing, China) to a stock solution of $200 \mathrm{mM}$ TMZ. Immediately before use in cell culture, the stock was diluted in media. Antibodies used for western blot analyses were obtained from Cell Signaling Technology (Danvers, MA, USA).

Cells and cell culture. Human glioma cell lines U87-MG and U251-MG were obtained from the American Type Culture 
Collection (ATCC; Manassas, VA, USA). TMZ-resistant cell lines U87-TMZ and U251-TMZ were obtained by culturing U87-MG or U251-MG cells with gradually increased doses (2-20 $\mu \mathrm{M})$ of TMZ for 4 months. All the cells were cultured in Dulbecco's modified Eagle's medium (DMEM) medium with $10 \%$ fetal bovine serum (FBS; Gibco, Vienna, Austria), 100 units/ml penicillin and $100 \mu \mathrm{g} / \mathrm{ml}$ streptomycin. All the cells were cultured at $37^{\circ} \mathrm{C}$ in an atmosphere comprising $95 \%$ air and $5 \% \mathrm{CO}_{2}$.

Real-time-PCR (RT-PCR). Cellular RNA was isolated by TRIzol reagent according to the manufacturer's instructions. Briefly, the DNA was removed from the samples using DNase treatment (DNA-free kit; Ambion-Applied Biosystems, Carlsbad, CA, USA) and cDNA was synthesized from the purified RNA using Moloney murine leukemia virus reverse transcription kit (Promega, Madison, WI, USA). Primers for human PomGnT1 were: F, 5'-GCCAAGTTTGCTGTGGTT CTGG-3' and R, 5'-CTGGTCATTCCAGGCAGAGATG-3'. Actin primer sets were used to produce a normalization control. Real-time PCR was carried out in triplicate with the SYBR-Green PCR Master Mix (Applied Biosystems, Foster City, CA, USA) and a 7900HT Fast Real-Time PCR machine (Applied Biosystems).

Western blot analysis. RIPA buffer in the presence of protease inhibitor cocktail and phosphorylation inhibitor cocktail were used to extract total protein. Appropriate mount protein was loaded into 8-10\% SDS-polyacrylamide gel and transferred onto a nitrocellulose membrane (Millipore, Billerica, MA, USA). Primary antibodies were incubated overnight and secondary antibodies were incubated for $1 \mathrm{~h}$ at the appropriate dilutions. The signal was observed and developed with Kodak film by exposure to enhanced chemiluminescence (ECL) plus western blotting detection reagents (Amersham Biosciences, Piscataway, NJ, USA). Western blot analysis was performed with antibodies against PomGnT1, TCF8, vimentin, $\beta$-catenin, Slug and actin was used as control.

Lentivirus mediated shRNA gene knockdown PomGnTl. The stable knockdown PomGnT1 cell lines were generated by transduction a lentiviral-mediated expression siRNA specific target of PomGnT1. Lentivirus containing the PomGnT1specific shRNAs (shRNA sequences targeting PomGnT1 was: SH1: 5'-GCCATTGAGCTCAGCAGAAGA-3'; SH2: 5'-GCA TCCAGCATACTCCCATCA-3'; SH3: 5'-AGGAGGAGCTTG AGCCCAA-3'; SH4: 5'-GGAGAAAGATGATGACTTC-3') were purchased from Shanghai Hanyu Biotechnology Co., Ltd. (Shanghai, China). The PomGnT1-specific shRNA lentiviral particles infected U87-TR and U251-TR cells with $10 \mu \mathrm{g} / \mathrm{ml}$ polybrene for $12 \mathrm{~h}$. Afterwards, the medium containing viral particles was removed and replaced with fresh medium. One 'non-target' construct containing an shRNA sequence that did not target any known human gene was transduced separately into U87-TMZ or U251-TMZ cells to serve as a scrambled negative control. After $72 \mathrm{~h}$, the cells were harvested, and the knockdown efficiency was tested by realtime PCR and western blot analysis. Transduced cell lines were named U87-TMZ PomGnT1-KD and U87-TMZ NC, and U251-TMZ PomGnT1-KD and U251-TMZ NC.
Lentivirus mediated overexpression of PomGnT1. The stable overexpression of PomGnT1 cell lines were generated by transduction a lentiviral-mediated overexpression PomGnT1. An oligonucleotide coding for PomGnT1 (NM_017739) was cloned into the plasmid murine stem cell virus (pMSCV)-puro retroviral vector (Clontech Laboratories, Inc., Mountain View, CA, USA) with which 293 T cells were transfected along with packaging plasmids pMD.env and pMD.gag.-pol by Shanghai Hanyu Biotechnology. The produced retroviruses were used to infect U87-MG and U251-MG cells, which were further selected in the presence of $1.0 \mathrm{mg} / \mathrm{ml}$ puromycin to establish a cell line with stable PomGnT1 overexpression (U87-PomGnT1-OE or U251-PomGnT1-OE). Cells infected with empty vector (EV) pMSCV-puro derived retroviruses were used as control (U87-NC or U251-NC). After $72 \mathrm{~h}$, the cells were harvested and the overexpression efficiency was tested by real-time PCR and western blot analysis.

Growth inhibition studies. TMZ-sensitive and -resistant glioma cells were plated in 96-well white plates at $5 \times 10^{3}$ cells/well, and TMZ (ranging from 200 to $1012.5 \mu \mathrm{M}$ ) was added and incubated for $48 \mathrm{~h}$. A Cell Counting kit-8 (CCK-8; Dojindo Laboratories, Kumamoto, Japan) was used to assess cell viability. Briefly, CCK-8 reagent was added (10 $\mu \mathrm{l} /$ well), cells were maintained for $2 \mathrm{~h}$, and the absorbance was read under a microplate reader (SpectraMax M5; Molecular Devices, Sunnyvale, CA, USA) at $450 \mathrm{~nm}$. All half-maximal inhibitory concentration $\left(\mathrm{IC}_{50}\right)$ values were determined using GraphPad Prism 5 software.

Flow cytometric analysis. Apoptosis was determined by translocation of phosphatidylserine to the cell surface using an Annexin V-FITC and PI apoptosis detection kit (BD Biosciences, San Diego, CA, USA). TMZ-sensitive and -resistant cells were plated in 6 -well plates at a density of $5 \times 10^{5}$ cells/well and treated with TMZ for $48 \mathrm{~h}$. The cells were then harvested and washed twice in cold phosphate-buffered saline (PBS) and resuspended in Annexin V-FITC and PI for $30 \mathrm{~min}$ in the dark. Cell apoptosis was analyzed by using the CellQuest software on a FACSAria flow cytometer (BD Biosciences). Fluorescence was detected with an excitation wavelength of $480 \mathrm{~nm}$.

Statistical analysis. For quantitative data, all results are expressed as the mean \pm SD. Statistical significance between the groups was determined using the Student's t-test using the SPSS 18.0 (SPSS, Inc., Chicago, IL, USA). Each experiment was repeated at least three times. $\mathrm{P}<0.01$ was considered statistically significant.

\section{Results}

Upregulated PomGnT1 expression in TMZ-resistant GBM cells. TMZ is the primary and most promising therapeutic drug for GBM. In order to establish TMZ-resistant GBM cell lines, we cultured U87-MG and U251-MG cells with gradually increased doses $(2-20 \mu \mathrm{M})$ of TMZ for 4 months. The $\mathrm{IC}_{50}$ analysis results of U87-TMZ, U251-TMZ resistant cells and parental cells showed that the $\mathrm{IC}_{50}$ value of both $\mathrm{TMZ}$-resistant cells was increased almost 5-fold (Fig. 1A and B). We 

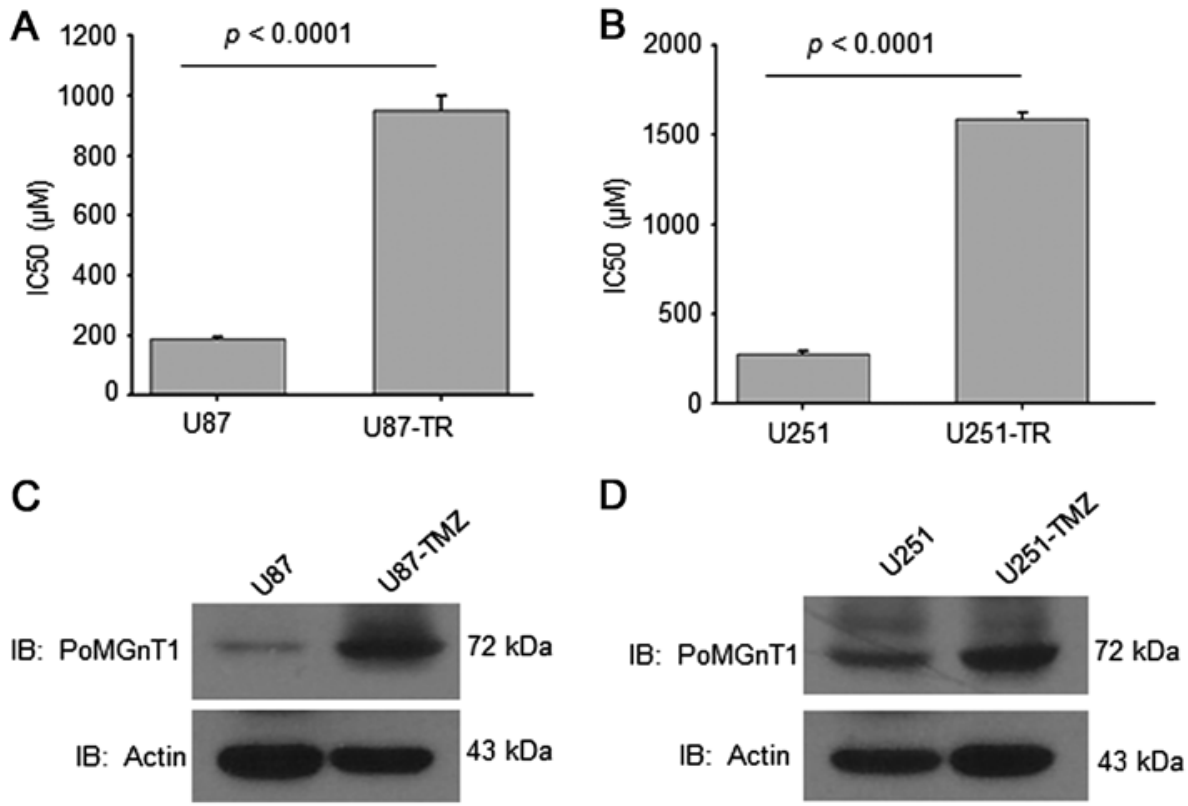

Figure 1. Establishment of TMZ-resistant GBM cells and expression of PomGnT1 in TMZ-resistant GBM cells. (A) IC 50 $_{0}$ analysis of TMZ-resistant U87-TMZ cells and parental U87-MG cells. (B) $\mathrm{IC}_{50}$ analysis of TMZ-resistant U251-TMZ cells and parental U251-MG cells. (C) Western blot analysis of PomGnT1 expression in U87-TMZ and parental U87-MG cells. (D) Western blot analysis of PomGnT1 expression in U251-TMZ and parental U251-MG cells.

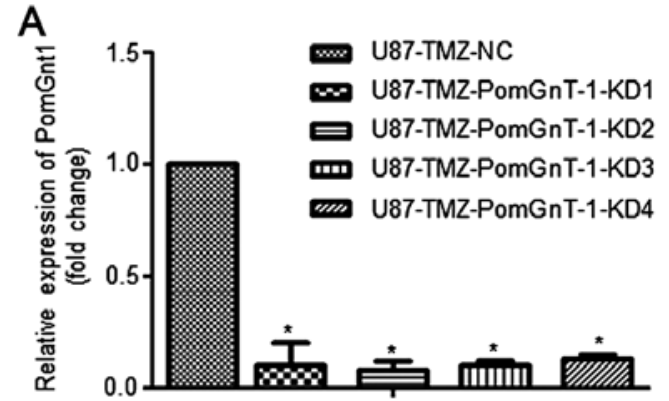

C

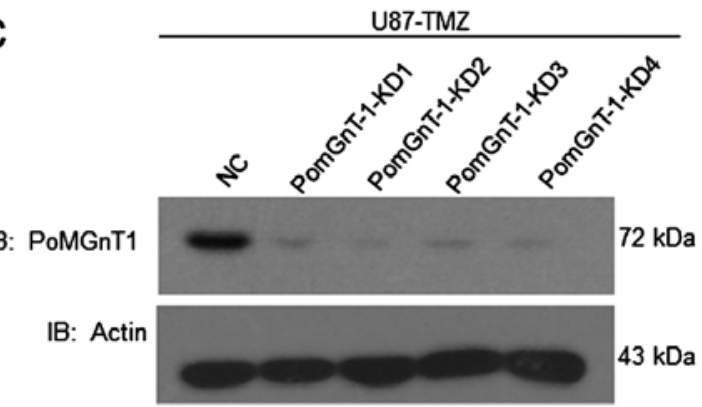

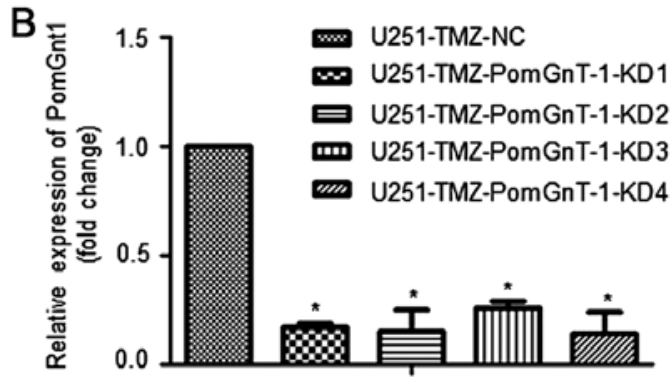

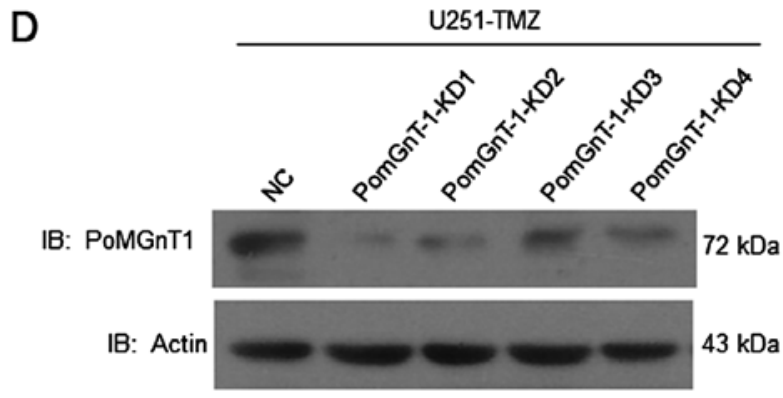

Figure 2. Suppression of PomGnT1 expression in TMZ-resistant GBM cells. (A and B) RT-PCR analysis of PomGnT1 mRNA levels in TMZ-resistant U87-TMZ (A) and U251-TMZ cells (B). (C and D) Western blot analysis of PomGnT1 protein levels in TMZ resistant U87-TMZ (C) and U251-TMZ cells (D).

further examined the expression of PomGnT1 in parental TMZ-sensitive (U87-MG and U251-MG) and TMZ-resistant (U87-TMZ and U251-TMZ) cells. As shown in Fig. 1C and D, PomGnT1 expression was significantly elevated in TMZ-resistant cells compared with matched parental sensitive cells. These data suggest that PomGnT1 expression might be associated with TMZ-resistance in GBM cells.

Suppression of PomGnT1 decreased $I C_{50}$ values for $T M Z$ and enhanced apoptosis of TMZ-resistant GBM cells.
Using lentivirus mediated shRNA gene knockdown system, we knocked down PomGnT1 expression in U87-TMZ and U251-TMZ cells. From the mRNA level, $>90 \%$ of PomGnT1 expression was decreased. The protein level of PomGnT1 was also decreased suggesting the high efficiency of shRNA knockdown system (Fig. 2). Cells were passaged upon reaching $80 \%$ confluence. Then, we treated PomGnT1 knocked-down U87-TMZ and U251-TMZ cells with TMZ (200-1350 $\mu \mathrm{M})$ for $48 \mathrm{~h}$ and detected the change of cell viability. As shown in Fig. 3, knockdown of PomGnT1 significantly reduced 

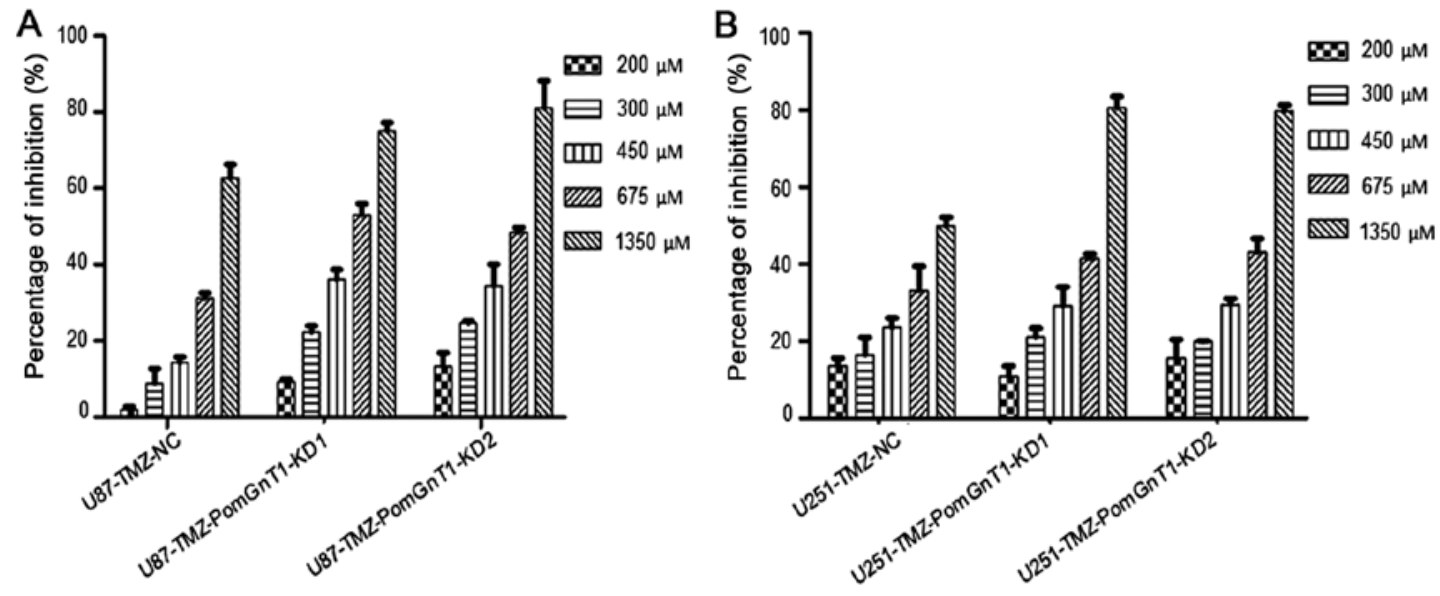

Figure 3. Knockdown of PomGnT1 in TMZ-resistant cell lines decreased IC $_{50}$ values for TMZ. (A) Knockdown of PomGnT1 reduced the viability of U87-TMZ cell in a dose-dependent manner. (B) Knockdown of PomGnT1 reduced the viability of U251-TMZ cells in a dose-dependent manner.
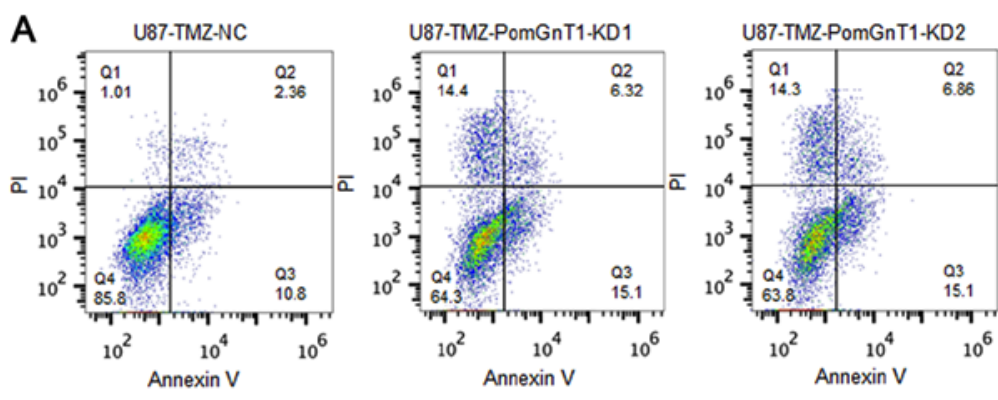

B
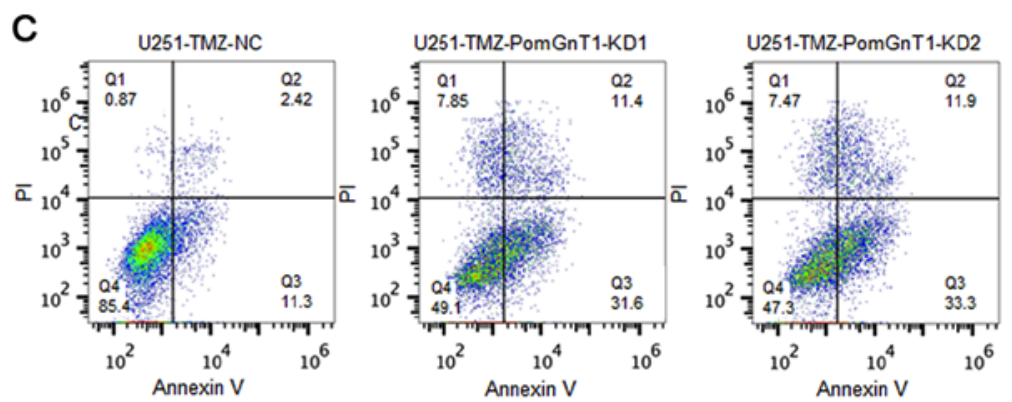

Figure 4. Suppression of PomGnT1 enhances apoptosis of TMZ-resistant GBM cells. (A and B) Knockdown of PomGnT1 increased apoptosis in U87-TMZ cells after treated with TMZ compared with matched parental negative cells. (C and D) knockdown of PomGnT1 induced apoptosis in U251-TMZ cells after treated with TMZ compared with matched parental negative cells.

Table I. $\mathrm{IC}_{50}$ values of U87-TMZ and U251-TMZ cells in response to TMZ.

\begin{tabular}{cccccccc}
\hline & \multicolumn{3}{c}{ U87-TMZ } & & \multicolumn{3}{c}{ U251-TMZ } \\
\cline { 2 - 3 } \cline { 7 - 8 } & NC & PomGnT1-KD1 & PomGnT1-KD2 & & NC & PomGnT1-KD1 & PomGnT1-KD2 \\
\hline $\mathrm{IC}_{50}(\mu \mathrm{M})$ & $1022.00 \pm 11.53$ & $643.67 \pm 7.77^{\mathrm{a}}$ & $632.00 \pm 10.54^{\mathrm{a}}$ & & $1322.67 \pm 16.17$ & $714.33 \pm 13.20^{\mathrm{a}}$ & $704.00 \pm 12.29^{\mathrm{a}}$ \\
\hline
\end{tabular}

Briefly, using lentivirus mediated shRNA gene knockdown system, resistant cells were seeded in 96-well plates, and treated with increasing

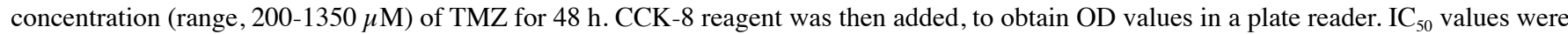

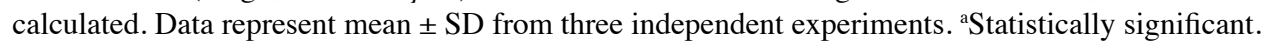

the viability of TMZ-resistant glioma cell lines, in a dosedependent manner. The $\mathrm{IC}_{50}$ value of $\mathrm{TMZ}$ for U87-resistant cells was $1033 \mu \mathrm{M}$ and for U251-resistant cells was $1308 \mu \mathrm{M}$.
However, the $\mathrm{IC}_{50}$ value of TMZ for U87-TMZ and U251-TMZ was decreased significantly after knockdown of PomGnT1 (Table I). Hence, there was a significant decrease in $\mathrm{IC}_{50}$ value 
Table II. $\mathrm{IC}_{50}$ values of $\mathrm{U} 87$ and $\mathrm{U} 251$ cells in response to TMZ.

\begin{tabular}{|c|c|c|c|c|}
\hline & \multicolumn{2}{|c|}{ U87 } & \multicolumn{2}{|c|}{$\mathrm{U} 251$} \\
\hline & $\mathrm{NC}$ & PomGnT1-OE & $\mathrm{NC}$ & PomGnT1-OE \\
\hline $\mathrm{IC}_{50}(\mu \mathrm{M})$ & $223.13 \pm 9.73$ & $906.37 \pm 7.75^{a}$ & $361.33 \pm 10.26$ & $953.67 \pm 14.84^{\mathrm{a}}$ \\
\hline
\end{tabular}
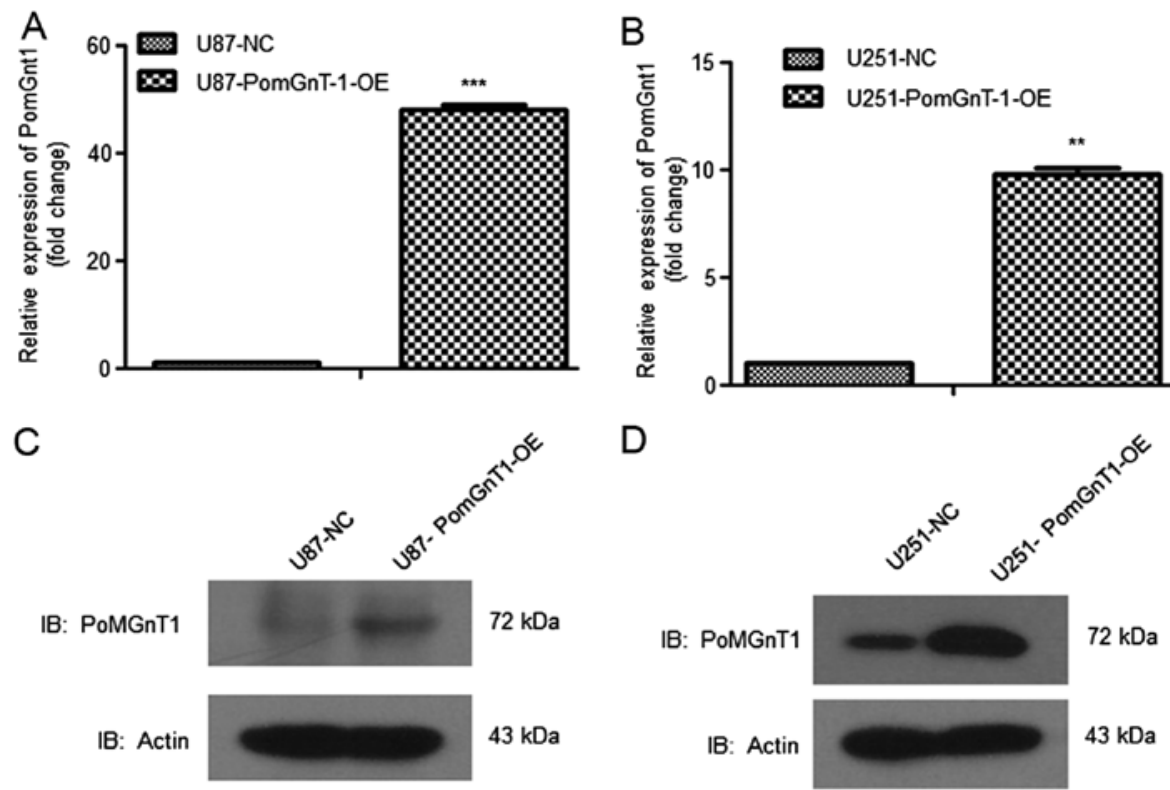

Figure 5. Overexpression of PomGnT1 in GBM cells. (A and B) RT-PCR analysis of PomGnT1 mRNA levels in control and PomGnT1 overexpressing cells. (C and D) Western blot analysis of PomGnT1 protein levels in control and PomGnT1 overexpressing cells.
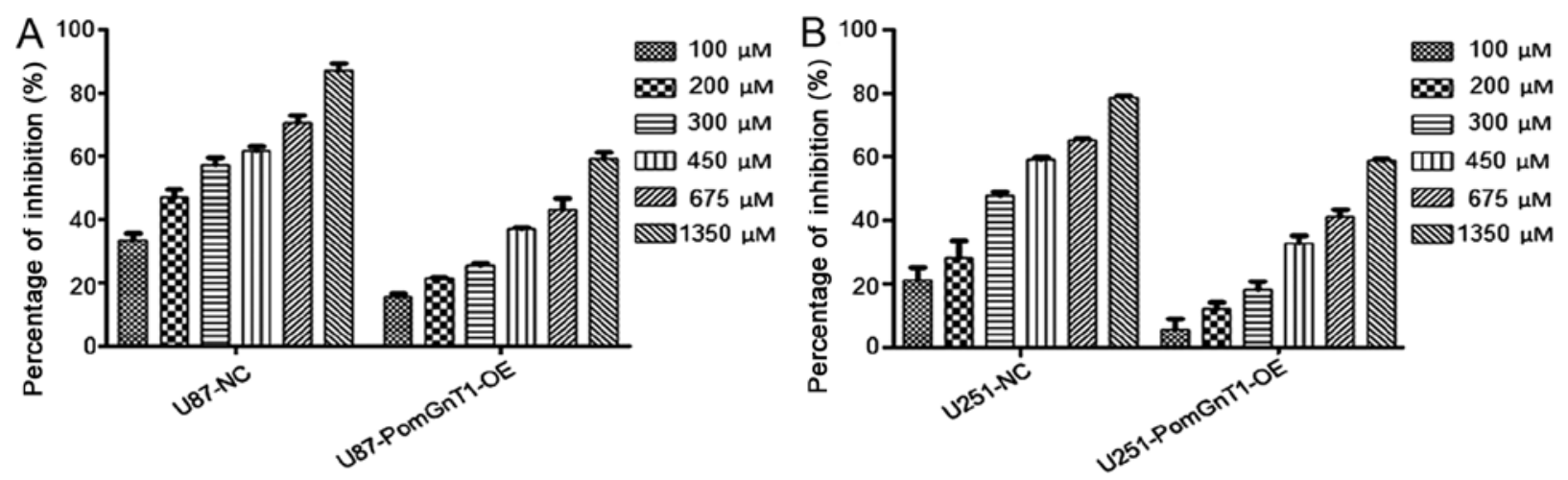

Figure 6. (A) Overexpression of PomGnT1 in TMZ-sensitive GBM cells increased $\mathrm{IC}_{50}$ values for TMZ. Overexpression of PomGnT1 increased the viability of U87-MG cells in a dose-dependent manner. (B) Overexpression of PomGnT1 increased the viability of U251-MG cells in a dose-dependent manner.

for TMZ-resistant GBM cells with knockdown of PomGnT1 compared with matched parental negative cells. Through the flow cytometric analysis, we found that knockdown of PomGnT1 significantly induced apoptosis in TMZ-resistant GBM cells after treated with TMZ compared with matched parental negative cells (Fig. 4). These results indicated that loss of PomGnT1 suppressed TMZ-resistant GBM cell survival mainly by induction of apoptosis after treated with TMZ.
Overexpression of PomGnTl increases $I_{50}$ values for $T M Z$ and reduced apoptosis of TMZ sensitive cells. Using lentivirus mediated gene overexpression system, we overexpressed PomGnT1 in U87 and U251 cells. From the mRNA level, PomGnT1 expression was increased $>10$-fold (Fig. 5A). The protein level of PomGnT1 was also increased suggesting the high efficiency of gene overexpression system (Fig. 5B). Then, we treated PomGnT1 overexpressed U87-MG and U251-MG 
A
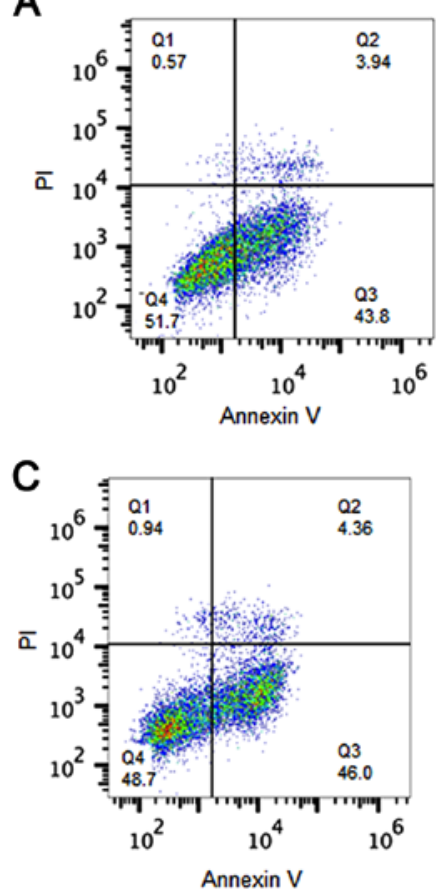
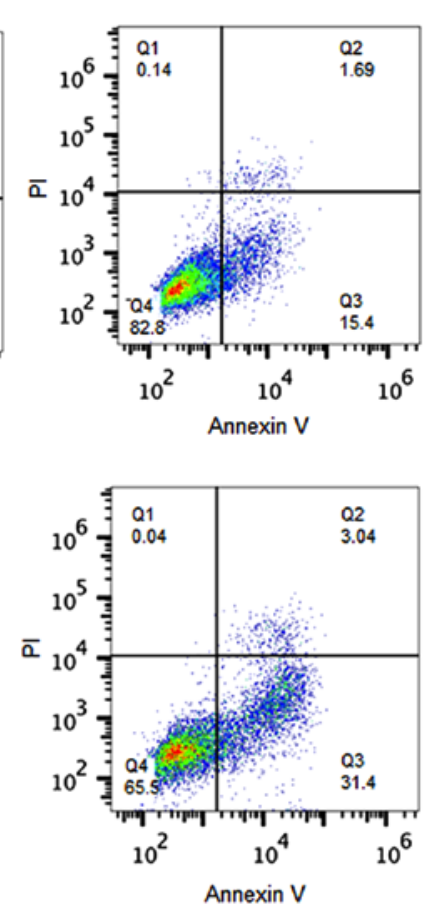

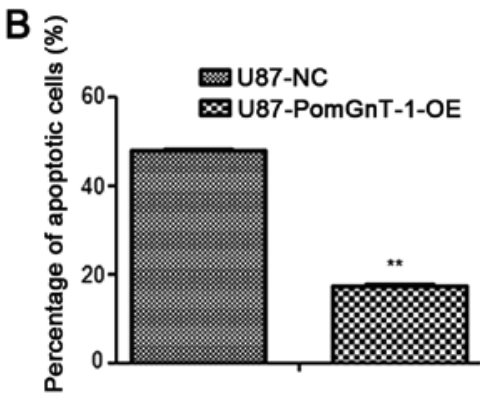

D

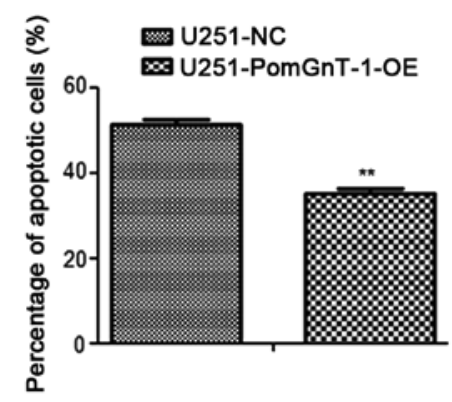

Figure 7. Overexpression of PomGnT1 reduced apoptosis of TMZ sensitive GBM cells. (A and B) The percentage of apoptotic PomGnT1 overexpressed in U87-MG cells was decreased after treated with TMZ compared with matched parental negative cells. (C and D) The percentage of apoptotic PomGnT1 overexpressed U251-MG cells was decreased after treated with TMZ compared with matched parental negative cells.
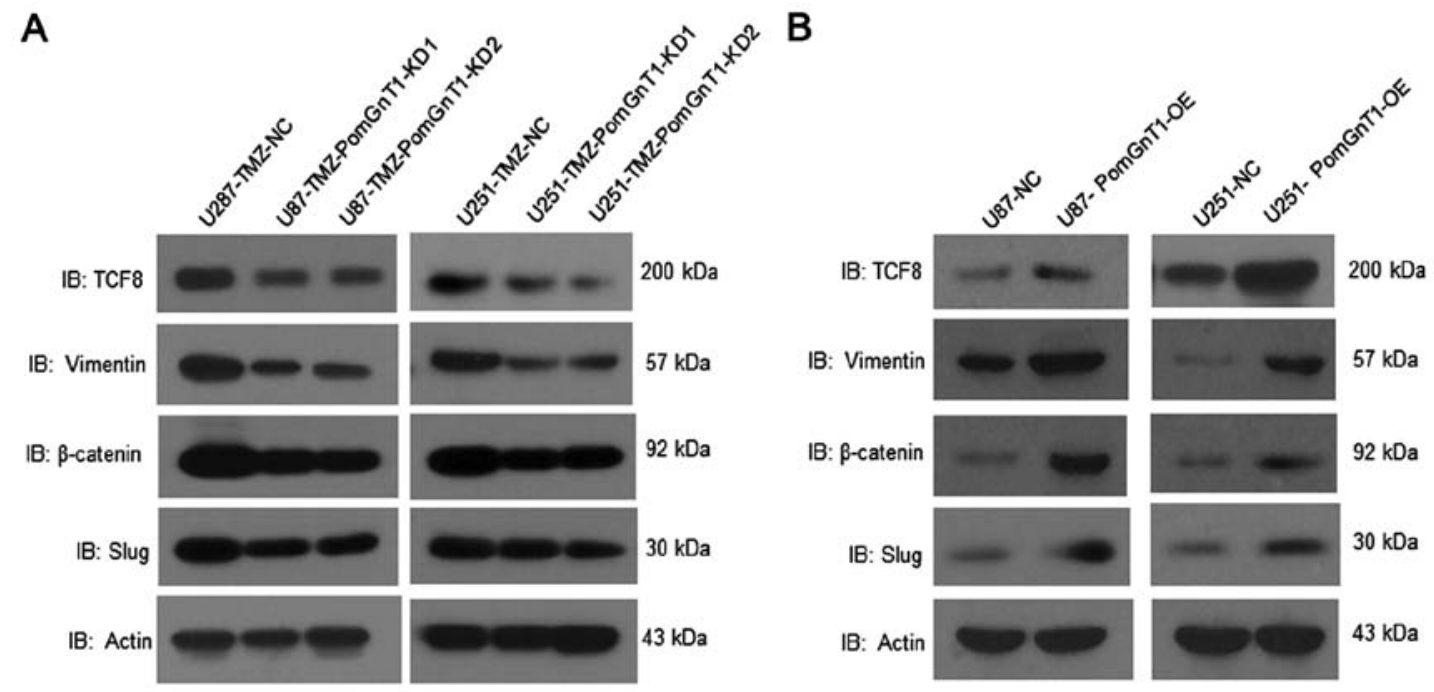

Figure 8. PomGnT1 regulates the expression of EMT markers in TMZ-resistant and TMZ-sensitive GBM cells after treated with TMZ. (A) Knockdown of PomGnT1 significantly inhibited expression of TCF8, vimentin, $\beta$-catenin and Slug in protein level in TMZ-resistant cells compared with matched parental negative cells. (B) Overexpression of PomGnT1 significantly up-regulated expression of TCF8, vimentin, $\beta$-catenin and Slug in protein level in TMZ-sensitive cells compared with matched parental negative cells.

cells with TMZ (100-1350 $\mu \mathrm{M})$ for $48 \mathrm{~h}$ and detected the change of cell viability. As shown in Fig. 6, overexpression of PomGnT1 increased the viability of TMZ-sensitive lines, in a dose-dependent manner. The $\mathrm{IC}_{50}$ value of $\mathrm{TMZ}$ for U87-MG was $227.4 \mu \mathrm{M}$ and for U251-MG was $364.1 \mu \mathrm{M}$. However, the $\mathrm{IC}_{50}$ value of TMZ for U87-MG and U251-MG was increased significantly after overexpression of PomGnT1 (Table II). Hence, there was a significant increase in $\mathrm{IC}_{50}$ value for TMZ-sensitive GBM cells with overexpression of PomGnT1 compared with matched parental negative cells. Through the flow cytometric analysis, we found that the percentage of apoptotic cells were decreased in TMZ-sensitive PomGnT1 overexpressed glioma cells after treated with TMZ compared with matched parental negative cells (Fig. 7). These results highlighted the importance of PomGnT1 in the progression of TMZ in treatment of TMZ-sensitive glioma cells.

PomGnT1 regulates the expression of EMT makers in TMZ-resistant GBM cells. To find the underlying molecular changes regulated by PomGnT1 in TMZ-resistant GBM 
cells, we observed the effect of PomGnT1 on TCF8, vimentin, $\beta$-catenin, Slug expression in TMZ-resistant and TMZ-sensitive GBM cells after treated with TMZ. As shown in Fig. 8, knockdown of PomGnT1 significantly inhibited expression of TCF8, vimentin, $\beta$-catenin and Slug in protein level in TMZ-resistant cells compared with matched negative control cells. Whereas, overexpression of PomGnT1 significantly elevated expression of TCF8, vimentin, $\beta$-catenin and Slug in protein level in TMZ-sensitive cells compared with matched negative control cells. These results indicate that PomGnT1-enhanced temozolomide resistance might partially be through activating epithelial-mesenchymal transition signaling in glioblastoma.

\section{Discussion}

The most challenging obstacle in the treatment of GBM is tumor recurrence (26-28). To date, because the recurring tumors are usually TMZ-resistant, there are only a few options for recurrent GBM and even these therapies have limited success $(29,30)$. Hence, strategies to neutralize and overcome chemo-resistance require thorough understanding of the diverse concepts, and are a significant unmet need in GBM therapy $(31,32)$. The present study explored the possibility of PomGnT1 in the chemo-resistance of TMZ in GBM cells. Elevated expression of PomGnT1 was detected in TMZ-resistant GBM cells compared to TMZ-sensitive GBM cells, indicating that PomGnT1 might be a new biomarker for TMZ treatment.

Moreover, knockdown of PomGnT1 in both TMZ resistant GBM cells decreased $\mathrm{IC}_{50}$ values for TMZ and enhanced apoptosis of GBM cells. On the contrary, overexpression of PomGnT1 in parental sensitive GBM cells increased $\mathrm{IC}_{50}$ values for TMZ and reduced apoptosis of GBM cells. The study revealed novel functions of PomGnT1 and indicated that PomGnT1 might be one of the major determinants conferring $\mathrm{TMZ}$ resistant properties in GBM.

We suggest that PomGnT1 influences the TMZ resistance as follows: Protein levels of EMT markers including TCF8, vimentin, $\beta$-catenin and Slug were changed with PomGnT1 expression, suggesting that PomGnT1 might be regulating TMZ resistance via modulating the EMT pathway. Cadherins, which are associated with cytoskeleton, epithelial-mesenchymal-transition as well as chemotherapy, have been found as substrates of PomGnT1 (33-35), indicating that PomGnT1 might influence TMZ resistance through the glycosylation modification of cadherins.

Taken together, our data revealed the importance of PomGnT1 in TMZ resistance in GBM and found a mechanistic pathway of PomGnT1 mediated EMT signaling. These studies demonstrate that PomGnT1 may be the focus of future research for treatment of recurrent TMZ-resistant GBM.

\section{Acknowledgements}

The present study was funded by the National Natural Science Foundation of China (no. 81502146), the Shanghai Municipal Commission of Health and Family Planning (no. 20154Y0067) and the Shanghai Jiaotong University Affiliated First People's Hospital (no. 06N1503016).

\section{References}

1. Wen PY and Kesari S: Malignant gliomas in adults. N Engl J Med 359: 492-507, 2008.

2. Jemal A, Bray F, Center MM, Ferlay J, Ward E and Forman D: Global cancer statistics. CA Cancer J Clin 61: 69-90, 2011.

3. Schwartzbaum JA, Fisher JL, Aldape KD and Wrensch M: Epidemiology and molecular pathology of glioma. Nat Clin Pract Neurol 2: 494-503, 2016.

4. Wilson CB: Glioblastoma: The past, the present, and the future. Clin Neurosurg 38: 32-48, 1992.

5. Glas M, Rath BH, Simon M, Reinartz R, Schramme A, Trageser D, Eisenreich R, Leinhaas A, Keller M, Schildhaus HU, et al: Residual tumor cells are unique cellular targets in glioblastoma. Ann Neurol 68: 264-269, 2010.

6. Grzmil M, Seebacher J, Hess D, Behe M, Schibli R, Moncayo G, Frank $\mathrm{S}$ and Hemmings BA: Inhibition of MNK pathways enhances cancer cell response to chemotherapy with temozolomide and targeted radionuclide therapy. Cell Signal 28: 1412-1421, 2016.

7. Lorenzetti M, Motta F, Campanella R, Bauer D, Assi A, Arienta C, Gaini SM and Caroli M: Adjuvant temozolomide chemotherapy for treatment of papillary tumor of the pineal region. World Neurosurg 76: 160-163, 2011.

8. Pace A, Vidiri A, Galiè E, Carosi M, Telera $\mathrm{S}$, Cianciulli AM, Canalini P, Giannarelli D, Jandolo B and Carapella CM: Temozolomide chemotherapy for progressive low-grade glioma: Clinical benefits and radiological response. Ann Oncol 14: 1722-1726, 2003.

9. Sarkaria JN, Kitange GJ, James CD, Plummer R, Calvert H, Weller M and Wick W: Mechanisms of chemoresistance to alkylating agents in malignant glioma. Clin Cancer Res 14: 2900-2908, 2008.

10. Segal MS, Goldstein MM and Attinger EO: The use of noscapine (narcotine) as an antitussive agent. Dis Chest 32: 305-309, 1957.

11. St-Coeur PD, Poitras JJ, Cuperlovic-Culf M, Touaibia M and Morin P Jr: Investigating a signature of temozolomide resistance in GBM cell lines using metabolomics. J Neurooncol 125: 91-102, 2015.

12. Akasaka-Manya K, Manya H, Kobayashi K, Toda T and Endo T: Structure-function analysis of human protein O-linked mannose beta1,2-N-acetylglucosaminyltransferase 1, POMGnT1. Biochem Biophys Res Commun 320: 39-44, 2004.

13. Lan J, Guo P, Chen M, Wu B, Mao Q and Qiu Y: O-linked mannose $\beta-1,2-N$-acetylglucosaminyltransferase 1 correlated with the malignancy in glioma. J Craniofac Surg 24: 1441-1446, 2013.

14. Lan J, Guo P, Lin Y, Mao Q, Guo L, Ge J, Li X, Jiang J, Lin X and Qiu Y: Role of glycosyltransferase PomGnT1 in glioblastoma progression. Neuro-oncol 17: 211-222, 2015.

15. Abbott KL, Troupe K, Lee I and Pierce M: Integrin-dependent neuroblastoma cell adhesion and migration on laminin is regulated by expression levels of two enzymes in the O-mannosyllinked glycosylation pathway, PomGnT1 and GnT-Vb. Exp Cell Res 312: 2837-2850, 2006.

16. Abbott KL, Troupe K, Matthews RT and Pierce M: GnT-Vb expression increases O-mannosyl-linked HNK-1 epitope leading to changes in neuronal cell adhesion and migration. Glycobiology 16: 1111-1111, 2006.

17. Miller MR, Ma D, Schappet J, Breheny P, Mott SL, Bannick N, Askeland E, Brown J and Henry MD: Downregulation of dystroglycan glycosyltransferases LARGE2 and ISPD associate with increased mortality in clear cell renal cell carcinoma. Mol Cancer 14: 141, 2015.

18. Zeisberg M and Neilson EG: Biomarkers for epithelial-mesenchymal transitions. J Clin Invest 119: 1429-1437, 2009.

19. De Craene B and Berx G: Regulatory networks defining EMT during cancer initiation and progression. Nat Rev Cancer 13: 97-110, 2013.

20. Makker A and Goel MM: Tumor progression, metastasis and modulators of EMT in endometrioid endometrial carcinoma: An update. Endocr Relat Cancer 23: R85-R111, 2015.

21. Shen Y, Zhou J, Li Y, Ye F, Wan X, Lu W, Xie X and Cheng X: miR-375 mediated acquired chemo-resistance in cervical cancer by facilitating EMT. PLoS One 9: e109299, 2014.

22. Toge M, Yokoyama S, Kato S, Sakurai H, Senda K, Doki Y, Hayakawa Y, Yoshimura N and Saiki I: Critical contribution of MCL-1 in EMT-associated chemo-resistance in A549 non-small cell lung cancer. Int J Oncol 46: 1844-1848, 2015. 
23. Zou C, Lu Y, Mizokami A, Keller ET, Pienta KJ and Zhang J: CCL2 and interleukin-6 regulate EMT-mediated chemo-resistance in prostate cancer. Cancer Res 72 (Suppl): Abst 841-841, 2012.

24. Liang SQ, Marti TM, Dorn P, Froment L, Hall SR, Berezowska S, Kocher G, Schmid RA and Peng RW: Blocking the epithelialto-mesenchymal transition pathway abrogates resistance to anti-folate chemotherapy in lung cancer. Cell Death Dis 6: e1824, 2015.

25. Liang S, Marti TM, Dorn P, Froment L, Hall S, Berezowska S, Kocher G, Schmid RA and Peng R: 18P Epithelial-tomesenchymal transition (EMT) is required for resistance to anti-folate chemotherapy in lung cancer. J Thorac Oncol 11: (Suppl) S63, 2016.

26. Stupp R, Hegi ME, van den Bent MJ, Mason WP, Weller M, Mirimanoff RO and Cairncross JG; European Organisation for Research and Treatment of Cancer Brain Tumor and Radiotherapy Groups; National Cancer Institute of Canada Clinical Trials Group: Changing paradigms: an update on the multidisciplinary management of malignant glioma. Oncologist 11: 165-180, 2006

27. Dehdashti AR, Hegi ME, Regli L, Pica A and Stupp R: New trends in the medical management of glioblastoma multiforme: The role of temozolomide chemotherapy. Neurosurg Focus 20 : E6, 2006

28. Lu C and Shervington A: Chemoresistance in gliomas. Mol Cell Biochem 312: 71-80, 2008.

29. Kroemer G, Galluzzi L, Vandenabeele P, Abrams J, Alnemri ES, Baehrecke EH, Blagosklonny MV, El-Deiry WS, Golstein P, Green DR, et al; Nomenclature Committee on Cell Death 2009: Classification of cell death: Recommendations of the Nomenclature Committee on Cell Death 2009. Cell Death Differ 16: 3-11, 2009.
30. Vega EA, Graner MW and Sampson JH: Combating immunosuppression in glioma. Future Oncol 4: 433-442, 2008.

31. Zhu Z, Du S, Ding F, Guo S, Ying G and Yan Z: Ursolic acid attenuates temozolomide resistance in glioblastoma cells by downregulating $\mathrm{O}^{6}$-methylguanine-DNA methyltransferase (MGMT) expression. Am J Transl Res 8: 3299-3308, 2016.

32. Bobola MS, Kolstoe DD, Blank A, Chamberlain MC and Silber JR: Repair of 3-methyladenine and abasic sites by base excision repair mediates glioblastoma resistance to temozolomide. Front Oncol 2: 176, 2012.

33. McConkey DJ, Choi W, Marquis L, Martin F, Williams MB, Shah J, Svatek R, Das A, Adam L, Kamat A, et al: Role of epithelial-to-mesenchymal transition (EMT) in drug sensitivity and metastasis in bladder cancer. Cancer Metastasis Rev 28: 335-344, 2009.

34. She X, Yu Z, Cui Y, Lei Q, Wang Z, Xu G, Luo Z, Li G and Wu M: miR-181 subunits enhance the chemosensitivity of temozolomide by Rap1B-mediated cytoskeleton remodeling in glioblastoma cells. Med Oncol 31: 892, 2014.

35. Vester-Christensen MB, Halim A, Joshi HJ, Steentoft C, Bennett EP, Levery SB, Vakhrushev SY and Clausen H: Mining the O-mannose glycoproteome reveals cadherins as major O-mannosylated glycoproteins. Proc Natl Acad Sci USA 110: 21018-21023, 2013 . 